\title{
Informational Approach to the Content of Musical Theoretical Disciplines
}

\author{
Yatsentkovskaya Nina Anatolyevna ${ }^{1}$ \\ ${ }^{1}$ St. Petersburg Children's School of Art, The Herzen State Pedagogical University of Russia, St. Petersburg, \\ Russia \\ Abstract: The basic principles of the information approach to the content of the music-theoretical disciplines \\ are formulated, its basic concepts are reviewed and analyzed
}

Keywords: information object, model, playing field, mode, leading reference point, contact and distant intervals

Music as a form of communication has received a new vector of development with the advent of information and communication technologies both as a significant area of culture and as an educational area [3], [4]. In this regard, the sections of music education which, on the one hand, are directly related to the musical language mediated by musical writing system, and on the other are invariant (common) for musicians of all specialties, namely, the musical-theoretical disciplines require not only changes in the forms of organizing the educational process, but also introducing a new approach to the content itself.

The peculiarity of a performing musician's activity is the fact that it is realized within the framework of a given text in a strictly structured musical period of time. Necessary for a solo musician is the ability to quickly master, hold in memory and accurately reproduce a large piece of information (repertoire); in the case of collective interaction during ensemble, orchestral or choral performance necessary is the ability to reproduce or perform a written (musical) text. And if earlier such result was achieved through intensification of labor, many hours of training and "learning", then now, with the advent of information technologies, with new possibilities for storage, transferring and presenting musical information, it becomes available to replace "learning" with "understanding" in the section of music education that is not connected directly with mastering the musical instrument itself (voice in particular).

This requires a new, informational approach to the content of musical theoretical disciplines. The preconditions for its appearance can be found already in early works on the theory of music and professional music education featured by tendency to structurize and systematizes separate elements of the musical language. The basis, scientific background and practical forms of implementing informational approach were developed and described in detail in the works of Nina A. Berger [1], [2].

Let us highlight the main principles determining the informational approach to the content of musical theoretical disciplines in music education:

1. The basic elements of musical language are presented as informational and material objects and models.

2. A keyboard is presented as a unfolded material appearance model of a structured musical pitch level space.

3. The concept of leading and not leading reference points for the basic and high levels of musician's theoretical thinking is introduced.

4. The absolute pitch of a sound reflected through the clef system and the position of a note on the musical staff is replaced by an interval. It is a leading guideline when reading notes. Upon that, "division of intervals into contact (between adjacent sounds of a melody or chord) and distant (between beginnings and cadences of different melodic phrases, in a sequence, imitation)" is introduced".Thus, an interval in the form of a virtual model becomes an acoustic-muscular reference point for the actions of a musician. The types of pitch 
connections are revealed not only directly in the "materialized" intonation situation, but also indirectly, at distance.

5. Introducing a "prepared playing ground" of an instrument at the early stages allows creating adequate (basic) reactions to the musical text directly in the process of reading, forms auditory images corresponding to the graphic. At higher levels of thinking, a holistic sound space, structured according to the conditions of tonality or another pitch system, acts as a defined set in the role of a similar "playing ground". A rather numerous set of elements processed by means of operations similar to arithmetic operations ("build a chord", "build a scale", etc.) is replaced by a small number of principles for defining structures and a very limited number of actions similar to actions in set theory (intersection, union, difference and addition). The result is increased sizes of integrated information units available for functioning (for processes) of RAM (individual 7+ 2 elements).

6. The mode organization (the most specific category of music) is visualized and geometrically shaped through "potential musical space" of a keyboard (E. Nazaikinsky). In the definition of a mode an analogy with the theory of sets can also be drawn: "a group of sounds limited in some parameters, possessing the ability to unfold in sequence and in simultaneity and functioning as a holistic system in musician's thinking". This concept is true for the ancient Greek modes, the halftoneless pentatonic, medieval modes, and the centralized mode of harmonic triad, modes of O. Messiaen, D. Shostakovich, the series, the turnover matrix (according to I. Istomin), etc. Clear simple visual keyboard images having geometrical forms can be imprinted by consciousness in a single act of attention. Such images are associated with the auditory-muscular sphere more intensively than any other determinants, forming auditory images through the "readiness of hands" (S. Maltsev).

7. Melody and chord appear as material objects, structures organized in time and space, through which music realizes itself as an art form.

8. In melody there are three levels of structuring: the first level of discreteness is related to melodic line; it is formed by large syntactic units, easily distinguished by perception, limited by breath. The position of such a unit in the scale of a mode and its range create the outer spatial form (outline), the architecture of a melody. Melodic idioms, forming the essence of the inner structure of a melody, become "lexical" units of the second level of discreteness. At the third level of discreteness, attention is drawn to a separate sound as a constructive unit of a melodic line. On the basis of contact and remote repetitions, regularities are established in the pitch connection of melodic constructions.

9. Organization of all the three levels is divided into strict and free. Strict organization is defined as creating prerequisites for an unambiguous assessment of the pitch situation, its identification, and the ability to promptly reproduce its parameters. With free organization, evaluation of the pitch structure is carried out indirectly: either through harmony, or analytically (that is, with a delay).

10. Such a concept as intertextual analysis of melodic thematism is introduced. Both types of organization of musical substance are identified through it. The principles of strict organization are systematized and reflected in geometric images-schemes, and are confirmed, for example, by analysis of the topics of the fugues from the polyphonic cycles of I. Bach, G. Kirchhoff, D. Shostakovich, P. Hindemith, S. Slonimsky.

11. The position of a sound, synchronous with the points of the time scale, is recognized as the leading guideline of rhythmicality at a basic level of thinking. Such forms of interaction with music as rhythmophonics and rhythm graphics are developed and introduced. By analogy with the names of sounds, related to their position in the system of spatial coordinates (pitch on a musical staff), the criterion for naming of a rhythmic element becomes its position in the time scale. Modified rhythmic syllables allow most rhythmic formulas to give an individual phonetic embodiment corresponding to intonational meaning. Similarly to the identity of sound names of sounds that are separated from each other by an octave in the pitch system, the syllables "Ta, Ta" in rhythmophonics become temporary landmarks on the path of musical movement. They assume the function of organizing a time scale (large divisions of the "ruler"), becoming intonational accents. 
The emergence of new forms of the educational process organization, the possibility of equipping the workplace of each student with different sets of software and hardware systems (synthesizer, electronic keyboard instruments, music computer) create the need for a new, informational approach to content. In this way, the reciprocal integration of music and the non-musical types of activity (E. Kozyrev) that are close to it in terms of psychological parameters can be realized; music appears as an art that is not purely aural, but is based on sensations synesthesia (M. Karaseva, A. Merriam, V. Morozov, V. Sradzhev, O. Shulpyakov, V. Yushmanov), that is, it is an activity based on the work of three of the five sensory systems (hearing, vision and touch) that bring thinking to discrete measuring scales. In the process of playing music, the orientation of a person in sound musical space and time gets the same foundation as during orientation in ordinary (physical) space and time with the help of inherent and acquired abilities. A keyboard becomes both a visual model of musical space and a field for modeling. Sound and style banks of software and hardware systems become the material for realizing such modeling [5], [6], [7]. Thus, the sphere of communicativeness of the musical art itself is expanding.

\section{References}

[1] Berger N.A. Extended abstract of the doctoral dissertation on the topic "Theory of music in modern practice of playing music". Saratov, 2011. 45 p.

[2] Berger N.A. Piano keyboard as an information system. Bulletin of the Chelyabinsk State University, vol. 37, 2009 , no. 35, pp. 160-167.

[3] Belov, G. G., \& Gorbunova, I. B. (2016). "Music and Cybernetics”. Music and Time, no.11, pp. 25-32.

[4] Gorbunova, I. B. (2017). "Information technology in music and music education". The World of Science, Culture, Education, vol. 63, no. 2, $206-210$.

[5] Gorbunova, I. B. (2014). "Information technology in music and integrated model of semantic space". Scientifictechnical Bulletin of Saint-Petersburg State Polytechnic University. Humanities and Social Sciences, 4 (208), 152-161.

[6] Gorbunova I. B., Rodionov D. P., Romanenko, L. Yu. (2013). "Music computer technologies in formation of information competence of a modern musician". Scientific and Technical Statements of St. Petersburg State Polytechnic University. Humanities and Social Sciences, no. 1 (167), pp. 39-45.

[7] Gorbunova I. B. (2017). "Musical computer: modeling of the process of musical creativity". The world of Science, Culture and Education, no. 4 (65), pp. 145-148. 